\title{
Patterns of Sonographically Detectable Echogenic Foci in Pediatric Thyroid Carcinoma with Corresponding Histopathology: An Observational Study
}

\author{
(D). Erdem Toslak, (DB. Martin, DG.A. Barkan, (D) A.I. Kılıç, and (D).E. Lim-Dunham
}

\begin{abstract}
BACKGROUND AND PURPOSE: Small echogenic foci within pediatric thyroid nodules are commonly seen by ultrasound and are one of the features used to determine the level of suspicion for malignancy. These are sometimes termed "microcalcifications," but their relation with malignancy is controversial due to the lack of standard terminology. Our aim was to evaluate sonographic patterns of echogenic foci in malignant pediatric thyroid nodules and describe the distribution of corresponding psammoma bodies and other histopathologic findings in thyroidectomy specimens.
\end{abstract}

MATERIALS AND METHODS: Ultrasounds of 15 pathologically proved malignant thyroid nodules in children were retrospectively reviewed by 2 radiologists who separately classified echogenic foci into the 4 morphologic patterns described in the American College of Radiology Thyroid Imaging, Reporting and Data System and noted their presence and distribution. Interobserver agreement was assessed, and consensus was reached for nodules for which there was disagreement. Surgical pathology findings from thyroidectomy specimens were retrospectively reviewed for the presence and distribution of psammomatous and dystrophic/stromal calcifications and eosinophilic/sticky colloid. Ultrasound and histopathologic ratings were compared, and frequencies and percentages corresponding to observed agreement levels were calculated.

RESULTS: Interobserver agreement between radiologists' sonographic assessments for the presence and distribution of echogenic foci ranged from $53 \%$ to $100 \%$ for all categories. Punctate echogenic foci were present in all nodules, and macrocalcifications, in $27 \%$. Histopathology of the 15 nodules revealed that only 4 (27\%) had psammomatous calcifications, while $9(60 \%)$ had stromal calcifications and $8(53 \%)$ had sticky colloid.

CONCLUSIONS: Sonographically detectable echogenic foci in malignant pediatric thyroid nodules can be reliably classified on the basis of American College of Radiology Thyroid Imaging, Reporting and Data System, with punctate echogenic foci composing the most common subtype. These echogenic foci do not represent psammomatous calcifications most of the time; instead, more than half of the malignant thyroid nodules with echogenic foci contained stromal calcifications or sticky colloid.

ABBREVIATIONS: ACR TI-RADS = American College of Radiology Thyroid Imaging, Reporting and Data System; PTC = papillary thyroid carcinoma; US = ultrasound; USFNAB = US-guided fine-needle aspiration biopsy

$\mathbf{P}$ unctate echogenic foci of $<1 \mathrm{~mm}$ are commonly seen by ultrasound (US) in thyroid nodules. These are often termed "microcalcifications" and are relevant because their presence within a nodule is one of several US features known to increase the likelihood of malignancy in both the pediatric and adult popula-

Received April 24, 2017; accepted after revision August 13.

From the Departments of Radiology (I.E.T., J.E.L.-D.) and Pathology (G.A.B., A.I.K.), Loyola University Medical Center, Maywood, Illinois; and Clinical Research Office (B.M.), Loyola University Chicago Health Sciences Division, Loyola University Chicago Stritch School of Medicine, Maywood, Illinois.

Please address correspondence to Jennifer E. Lim-Dunham, MD, Department of Radiology, Loyola University Medical Center, 2160 S 1st Ave, Maywood, IL 60153; e-mail jlim2@lumc.edu

http://dx.doi.org/10.3174/ajnr.A5419 tions. ${ }^{1-4}$ Microcalcifications are generally assumed to represent psammoma bodies, which are considered specific for papillary thyroid carcinoma (PTC) but may also be found in follicular or medullary thyroid carcinoma and thyrotoxicosis. ${ }^{5}$ Nevertheless, many PTCs do not contain psammoma bodies, which are found in only $25 \%-37 \%$ of PTCs. ${ }^{5,6}$ Moreover, other histologic features such as dystrophic or stromal calcifications and eosinophilic or "sticky colloid" may be seen in PTC and other types of thyroid malignancies. ${ }^{5-7}$

The importance of sonographically detectable microcalcifications in thyroid nodules is controversial. ${ }^{3,8-12}$ This controversy may be partially due to lack of standard terminology and of proper subclassification of the calcifications on the basis of morphologic features. Several US categorization systems for echo- 

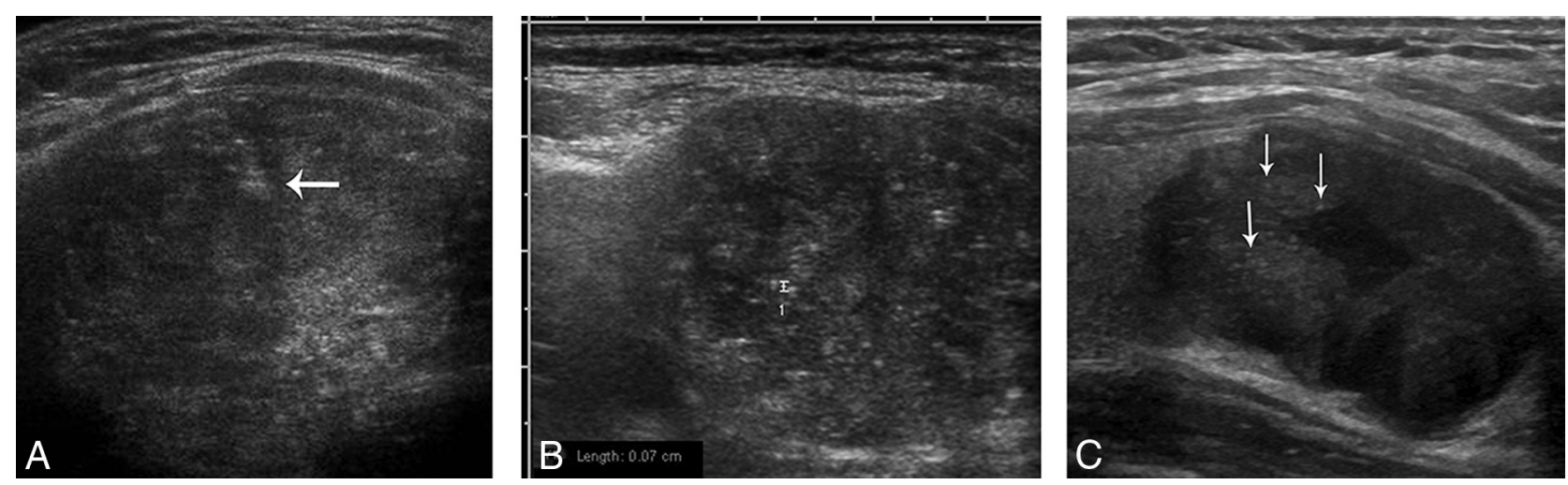

FIG 1. Typical sonography samples of categories of ACR TI-RADS echogenic foci and types of distribution. A, Macrocalcifications with posterior acoustic shadowing (arrow) in a left-sided 4.7-cm thyroid nodule in an 18-year-old girl with histologically proved papillary thyroid carcinoma. The distribution is classified as sparse. $B$, Punctate echogenic foci with small, $0.7-\mathrm{mm}$ comet-tail artifacts in the same patient as in $A$. The distribution is diffuse. Pathology revealed stromal calcifications and sticky colloid, both sparsely distributed, and the absence of psammomatous calcifications. C, Punctate echogenic foci with no posterior artifacts (arrows) in a left-sided 3.5-cm thyroid nodule in a 12-year-old girl with a histologically proved follicular variant of papillary thyroid carcinoma. The distribution is sparse. Histology revealed no corresponding calcifications or sticky colloid.

genic foci in thyroid nodules have been developed, with some studies showing that echogenic foci previously termed "microcalcifications" were present not only in malignant thyroid nodules but also in benign nodules. ${ }^{11,12}$ A recent study on adults that subclassified echogenic foci reported that what many authors called microcalcifications did not exclusively represent psammoma bodies, but rather other entities including stromal calcifications and sticky colloid. ${ }^{7}$ On the basis of these findings, the recently proposed American College of Radiology Thyroid Imaging, Reporting and Data System (ACR TI-RADS) terminology used to describe echogenic foci in thyroid nodules has removed the word "microcalcification" from its lexicon and replaced it with more precise descriptors. ${ }^{4}$

To our knowledge, no dedicated study has previously been undertaken in the pediatric population of patterns of sonographic echogenic foci and corresponding histopathology. We hypothesized that as in adults, echogenic foci in malignant thyroid nodules may not always represent psammomatous calcifications. Therefore, the aim of this observational investigation was to evaluate echogenic foci in malignant pediatric thyroid nodules according to ACR TI-RADS and to describe the distribution of corresponding psammoma bodies and other histopathologic findings in thyroidectomy specimens.

\section{MATERIALS AND METHODS \\ Ethics Statement}

This retrospective study was approved by the institutional review board of Loyola University Medical Center, and written informed consent was waived. Patient records and information were anonymized and randomized before analysis.

\section{Patient Selection}

The study cohort included 29 consecutive pediatric patients, 18 years of age and younger, who underwent total thyroidectomy for thyroid malignancy between 1996 and 2016. Ten patients were excluded due to the lack of preoperative US images, and 4 patients were excluded due to the lack of surgical specimens, leaving 15 patients composing the final study group.

\section{Ultrasonography and Image Analysis}

All individuals underwent diagnostic gray-scale ultrasonography performed on a variety of US systems (Acuson Sequoia 512, XP128 and Aspen, Siemens, Erlangen, Germany; and Logic E9, GE Healthcare, Milwaukee, Wisconsin) using high-frequency $(8-15 \mathrm{MHz})$ linear array transducers. Still and cine US images of the thyroid, including any focal nodules, were stored electronically in DICOM format on the PACS server. These images were independently evaluated by 2 radiologists (J.E.L.-D., I.E.T.), each with $>10$ years of experience and each blinded to the pathologic diagnosis. For nodules for which there was disagreement, consensus was reached in a separate session.

Echogenic foci in each nodule were classified into 1 of 4 morphologic categories on the basis of ACR TI-RADS lexicon descriptions ${ }^{4,13}$ : echogenic foci with large $\mathrm{V}$-shaped comet-tail artifacts measuring $>1 \mathrm{~mm}$; macrocalcifications, defined as coarse echogenic foci accompanied by acoustic shadowing; peripheral rim calcifications located along the margin of the nodule; and punctate echogenic foci with no or small $(<1-\mathrm{mm})$ comet-tail artifacts. Typical examples of each category found in our patient population are illustrated in Fig 1. Examples of echogenic foci with large comet-tail artifacts and peripheral calcifications were not included because these were not found in any of our patients. If a nodule contained $>1$ type of echogenic focus, all relevant categories were applied.

The distribution of the echogenic foci was then analyzed. Sparse was defined as 1-3 scattered echogenic foci; focal, as $>3$ foci in a local cluster; and diffuse, as innumerable echogenic foci scattered throughout the nodule, in accordance with the system described by Tahvildari et al. ${ }^{7}$ Typical examples of distribution are also demonstrated in Fig 1.

\section{Tissue Diagnosis}

Before thyroidectomy, 14 of the 15 nodules underwent US-guided fine-needle aspiration biopsy (USFNAB). The decision to proceed to thyroidectomy was made by the endocrine surgeon on the basis of USFNAB results for 12 patients. For the other 3 patients, including the patient who did not undergo USFNAB, thyroidec- 


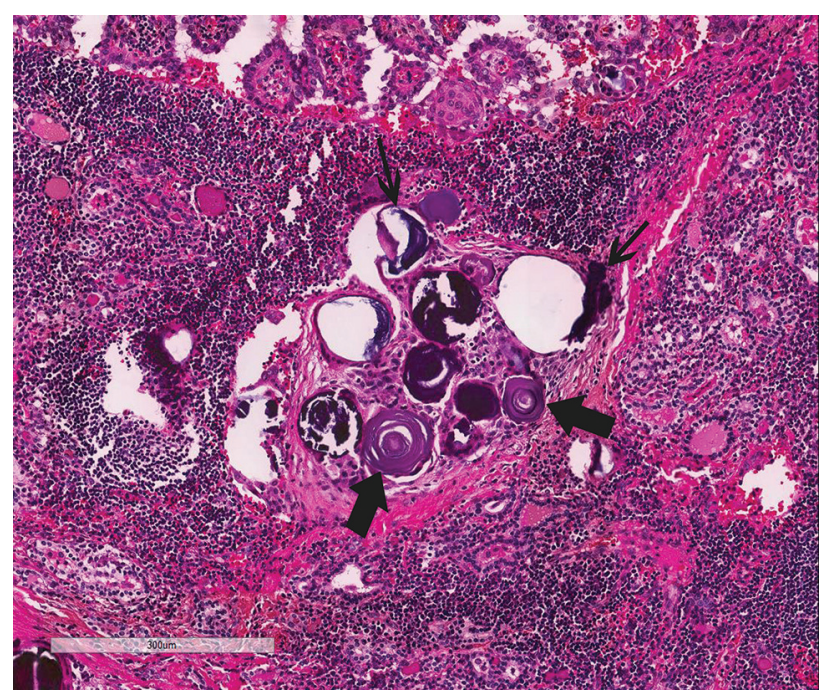

FIG 2. Examples of psammomatous (thick arrows) and stromal calcifications (thin arrows) from a thyroidectomy specimen of a 16-yearold girl showing papillary thyroid carcinoma in a background of lymphocytic thyroiditis (hematoxylin-eosin stain, 20X).

tomy was performed according to clinical and US findings. USFNAB cytology results were retrieved from the medical records; 9 (60\%) of the cytology slides were available for review by a board-certified pathologist (G.A.B.).

The Bethesda System for Reporting Thyroid Cytopathology was used to classify the thyroid nodules as follows: nondiagnostic, benign, atypia or follicular lesion of undetermined significance, follicular neoplasm/suspicious for follicular neoplasm, suspicious for malignancy, or malignant. ${ }^{14}$ Surgical pathology slides of the thyroidectomy specimens were retrospectively reviewed by the same pathologist. Care was taken to ensure that each surgical pathology sample corresponded in size and location to the designated nodule noted on the US evaluation. Histology samples were evaluated for the presence of psammomatous and stromal calcifications based on the previous descriptions in the literature, with a typical example demonstrated in Fig 2. ${ }^{6}$ The distribution of each type of calcification was characterized as sparse, focal, or diffuse. Sparse was defined as rare calcifications that were detected only on close screening; focal, as calcifications easily identified but localized to 1 area; and diffuse, as calcifications scattered throughout the tumor, as described by Tahvildari et al. ${ }^{7}$ Colloid, if present, was characterized as thin versus "sticky."

\section{Statistical Analysis}

Descriptive statistics included a demographic analysis by patient sex, age, histology outcome, and nodule size. Patient age and nodule size were expressed as medians and interquartile ranges due to the small sample size.

Weighted $\kappa$ coefficients were initially generated with Cicchetti-Allison weights to assess interobserver reliability between the 2 radiologists' ordinal ratings of the sonographic echogenic foci morphologic type and distribution of each nodule. However, the small sample size coupled with high levels of observed agreement ultimately precluded the use of $\kappa$ coefficients in the final analysis. Thus, frequencies and percentages corresponding to ob-
Table 1: Observed agreement between radiologists for presence and distribution of thyroid nodule echogenic foci ${ }^{a}$

\begin{tabular}{lcc}
\hline \multicolumn{1}{c}{ US Classification } & $\begin{array}{c}\text { Agreement for } \\
\text { Presence or } \\
\text { Absence (No.) (\%) }\end{array}$ & $\begin{array}{c}\text { Agreement for } \\
\text { Distribution } \\
\text { (No.) (\%) }\end{array}$ \\
\hline $\begin{array}{l}\text { Punctate echogenic foci } \\
\text { Echogenic foci with large }\end{array}$ & $15 / 15(100 \%)$ & $14 / 15(93 \%)$ \\
$\quad$ comet-tail artifacts & $11 / 15(73 \%)$ & $11 / 15(73 \%)$ \\
$\begin{array}{l}\text { Macrocalcifications } \\
\text { Peripheral rim calcifications }\end{array}$ & $8 / 15(53 \%)$ & $8 / 15(53 \%)$ \\
\hline
\end{tabular}

Note:-No. indicates number of nodules.

a The valid number of observations is 15 .

served agreement levels were reported for this observational study.

Radiologists' consensus ratings for the presence and distribution of echogenic foci of the various morphologic types on US were then compared with the observation of psammomatous calcifications, stromal calcifications, and sticky colloid on the surgical specimens. As before, these findings were presented as crosstabulations of patient counts due to the small sample size and substantial levels of observed agreement. All statistical analyses were conducted with SAS 9.4 (SAS Institute, Cary, North Carolina).

\section{RESULTS}

The 15 patients ( 14 female and 1 male) included in the study each had 1 thyroid nodule. The median age was 15 years (interquartile range, 13-18 years), and the median nodule size was $3.2 \mathrm{~cm}$ (interquartile range, $2.1-4.7 \mathrm{~cm}$ ). Of the 14 nodules that underwent USFNAB, results were classified according to the Bethesda System as malignant in $9(60 \%)$, follicular neoplasm/suspicious for follicular neoplasm in 3 (20\%), and benign in 2 (13\%). Diagnosis on thyroidectomy specimens was as follows: PTC in 10 (67\%), follicular variant of PTC in 1 (6.5\%), follicular thyroid carcinoma in 3 (20\%), and poorly differentiated carcinoma in 1 (6.5\%). Two cases reported as benign on cytology were identified on thyroidectomy specimens as PTC in one case and poorly differentiated carcinoma in the other. On retrospective review of the slides, this discrepancy was attributed to sampling error.

Observed agreement between the radiologists for the presence of echogenic foci ranged from $53 \%$ to $100 \%$, with agreement lowest for macrocalcifications and highest for punctate echogenic foci and peripheral rim calcifications (Table 1). Both raters agreed in 14 nodules (93\%) on the distribution of the punctate echogenic foci and agreed in all 15 nodules that peripheral rim calcifications were absent. There was a lower concordance for the macrocalcification subtype $(8 / 15,53 \%)$ (Table 1$)$.

The radiologists' consensus rating regarding the presence of echogenic foci revealed that all 15 nodules contained punctate echogenic foci, of which 14 were diffusely distributed. Four nodules $(27 \%)$ had macrocalcifications, of which 3 were sparsely distributed. Considering all patterns of echogenic foci and that some nodules contained $>1$ type of echogenic focus, there were 19 unique occurrences of echogenic foci; of these, 14 (74\%) were rated as diffusely distributed, and 4 (21\%), as sparsely distributed. There were no echogenic foci with large comet-tail artifacts or peripheral rim calcifications detected in any of the nodules.

Histologic examination revealed that 12 of the 15 (80\%) nod- 
Table 2: Thyroid nodule pathology as a function of US patterns ${ }^{a}$

\begin{tabular}{|c|c|c|c|c|}
\hline \multirow[b]{2}{*}{ Pathology Finding (No.) } & \multicolumn{2}{|c|}{$\begin{array}{l}\text { Punctate Echogenic } \\
\text { Foci (No.) }\end{array}$} & \multicolumn{2}{|c|}{ Macrocalcifications (No.) } \\
\hline & Present $(n=15)$ & Absent $(n=0)$ & Present $(n=4)$ & Absent $(n=11)$ \\
\hline Stromal calcification present (9) & $9(60 \%)$ & $0(0 \%)$ & $4(100 \%)$ & $5(45 \%)$ \\
\hline Stromal calcification absent (6) & $6(40 \%)$ & $0(0 \%)$ & $0(0 \%)$ & $6(55 \%)$ \\
\hline Psammomatous calcification present (4) & $4(27 \%)$ & $0(0 \%)$ & $1(25 \%)$ & $3(27 \%)$ \\
\hline Psammomatous calcification absent (11) & $11(73 \%)$ & $0(0 \%)$ & $3(75 \%)$ & $8(73 \%)$ \\
\hline Sticky colloid present (8) & $8(53 \%)$ & $0(0 \%)$ & $3(75 \%)$ & $5(45 \%)$ \\
\hline Sticky colloid absent (7) & $7(47 \%)$ & $0(0 \%)$ & $1(25 \%)$ & $6(55 \%)$ \\
\hline
\end{tabular}

Note:-No. indicates number of nodules.

a The valid number of observations is 15 .

ules had some type of calcification or sticky colloid, while 3 (20\%) nodules had none. Nine $(60 \%)$ nodules had stromal calcifications, of which 4 were diffusely distributed and 4 were sparsely distributed. All 4 (27\%) nodules that contained psammomatous calcifications were rated as diffusely distributed. Eight (53\%) nodules had sticky colloid, which was sparsely distributed in all. Considering all patterns of calcification or sticky colloid and that some nodules contained $>1$ type of abnormality, there were 21 unique occurrences; of these, 8 (38\%) were rated as diffusely distributed, and 12 (57\%), as sparsely distributed.

Radiologists' consensus ratings for the presence of sonographic echogenic foci were then compared with histologic findings (Table 2). Radiologists detected the punctate echogenic foci subtype in all 15 nodules. Histopathology revealed that among these 15 nodules, 9 (60\%) had stromal calcifications, $4(27 \%)$ had psammomatous calcifications, and $8(53 \%)$ had eosinophilic/ sticky colloid. Thus, most $(11 / 15,73 \%)$ patients determined to have punctate echogenic foci did not have psammomatous calcifications.

The subtype of macrocalcifications were less commonly seen by US $(4 / 15,27 \%)$ (Table 2). Pathology revealed that among these nodules, all $4(100 \%)$ had stromal calcification, 1 (25\%) had psammomatous calcifications, and $3(75 \%)$ had sticky colloid. Results confirmed the absence of psammomatous calcifications in 3 of the 4 (75\%) patients determined to have macrocalcifications.

Analysis of sonographic patterns by final pathologic diagnosis showed that all 10 PTC nodules were determined by US to have punctate echogenic foci, while 4 of them (40\%) had macrocalcifications. Of the 3 follicular thyroid carcinoma nodules, all had punctate echogenic foci.

Analysis of histologic findings segregated by final pathologic diagnosis showed that though all 4 psammomatous calcifications detected by histopathology were present in PTC nodules, of the 10 PTC nodules, most $(6 / 10,60 \%)$ lacked psammomatous calcifications. This was the case even though all 10 nodules were rated by radiologists' consensus as having punctate echogenic foci. Instead, $9(90 \%)$ of the PTC nodules were found to have stromal calcifications, and $6(60 \%)$, to have sticky colloid. Sticky colloid was found in 1 of the 3 follicular thyroid carcinoma nodules and in the single poorly differentiated carcinoma nodule. The nodule with the follicular variant of PTC did not have any type of calcification. None of the follicular thyroid carcinomas, poorly differentiated carcinomas, or follicular variant of PTC nodules had stromal or psammomatous calcifications.

\section{DISCUSSION}

As recommended by the 2015 American Thyroid Association Management Guidelines for Children with Thyroid Nodules and Differentiated Thyroid Cancer, small echogenic foci seen within thyroid nodules by US are one of the features used to determine the level of suspicion for malignancy. ${ }^{1}$ These are considered in conjunction with other features like margin irregularity and solid composition. ${ }^{3}$ These echogenic foci are often termed "microcalcifications," though exact definitions are often not stated or vary from study to study. ${ }^{3,8,10-12}$ Publications describing systems to standardize terminology include Beland et $\mathrm{al}^{11}$ and the ACR TIRADS, ${ }^{4,13}$ both of which subclassified echogenic foci into 4 patterns, and the study of Malhi et $\mathrm{al},{ }^{12}$ which classified echogenic foci into 5 patterns.

Our study used the ACR TI-RADS lexicon for subclassification of echogenic foci because the system is simple and widely accepted. We found high interrater observed agreements up to $100 \%$ for the presence of almost all echogenic foci subtypes, indicating that the application of TI-RADS was a reproducible method.

Psammoma bodies are lamellated calcifications that may be found in papillary types of tumors and are one of the features used to make the histologic diagnosis of PTC. ${ }^{6}$ This type of calcification is also important because PTC nodules that contain psammoma bodies are associated with lower disease-free survival rates, higher rates of lymph node metastasis, and a higher risk of pulmonary metastasis. ${ }^{6}$ Stromal calcifications without lamellated morphology did not have any impact on disease-free survival. ${ }^{6}$ Another diagnostic feature that may be found in up to $13 \%$ of PTCs is so-called "sticky colloid," which stains more densely and deeply eosinophilic than the typical "thin" or "watery" colloid, normally found in benign thyroid conditions. ${ }^{5}$

Our study revealed that even though most malignant pediatric nodules with punctate echogenic foci subtype were PTC, more than half $(11 / 15,73 \%)$ lacked psammomatous calcifications on pathologic examination. The absence of psammomatous calcifications by histology was also true for those with the macrocalcification subtype $(3 / 4,75 \%)$. We conclude that in children, sonographically detected punctate echogenic foci in malignant thyroid nodules do not always represent psammomatous calcifications; therefore, the term "punctate echogenic foci" may be more accurate than "microcalcification."

These results are like those from a recent study by Tahvildari et $\mathrm{al}^{7}$ of 29 adult PTC nodules with sonographically detected punctate echogenic foci classic for microcalcifications that showed that 
$6(21 \%)$ did not contain any calcifications by histology. Only 14 (48\%) contained psammomatous calcifications, either alone or in combination with coarse calcifications; therefore, in more than half (52\%), psammoma bodies were absent.

Of the pathologic features examined in our thyroid nodules, the most common was stromal calcifications, which were detected in $9 / 15(60 \%)$. Less commonly seen were sticky colloid in $8 / 15$ (53\%) and psammomatous calcifications in 4/15 (27\%). Tahvildari et $\mathrm{al}^{7}$ reported rates of $38 \%, 100 \%$, and $42 \%$, respectively, indicating a similar low percentage of nodules containing psammomatous calcifications. Our study suggests that in children, like adults, punctate echogenic foci in malignant nodules are more often associated with, and presumably sometimes represent, sticky colloid and stromal calcifications, rather than exclusively psammoma bodies. ${ }^{6,7}$ A possible explanation for this pathologic correspondence is that stromal calcifications and sticky colloid may cause US artifacts like those caused by psammomatous calcifications. Additionally, it has been proposed that in the absence of any pathologically proved calcifications or sticky colloid, such as observed in 3 (20\%) of our patients, the presence of tiny cysts beyond the resolution of US may cause specular reflection from the cyst walls, thus producing bright echogenic foci on US. ${ }^{7,12,15}$

In addition to the lack of US and histologic concordance for the presence of punctate echogenic foci, we also noted a mismatch in the distribution. Most of our sonographically detected echogenic foci were diffusely distributed, while less than half of the pathologically detected calcifications were diffusely distributed. Prior studies found a similar overestimation of diffuse distribution of echogenic foci on US compared with histology. ${ }^{7}$ The discrepancy might be partly due to inherent technical differences between histology and radiology. During US examination, the entirety of a nodule can be visualized in a global fashion on a cine loop sweep, whereas histologic examination is limited to selected sections of surgical specimens.

All 15 nodules, which were all malignant, contained punctate echogenic foci. The observed rate of this pattern in children agrees with the rate of $96 \%$ in adults reported previously. ${ }^{12}$ Past studies concerning US of pediatric thyroid nodules reported rates of microcalcifications in malignant nodules ranging from $25 \%$ to $44 \%$, which are much lower than the rates found in our study. ${ }^{10,16}$ This discrepancy might be due to variation in histologic types in the study populations or sample size. Additionally, the US resolution and/or definition of what constituted microcalcification may have differed between the studies because the type of US equipment and terminology used were not always explicitly stated. ${ }^{10,16}$

Because psammomatous calcifications are generally associated with the PTC type of thyroid malignancy, we also analyzed our data for PTC nodules only. Ten nodules (67\%) were PTC, which is different from the past literature indicating that PTC composes $92 \%-100 \%$ of pediatric thyroid malignancies. ${ }^{10,16}$ Of our PTC nodules, psammomatous calcifications were seen in only $40 \%$. This number agrees with the rates of $25 \%-37 \%$ reported in the adult literature and argues against the assumption that psammomatous calcifications are a specific feature of PTC. ${ }^{5,6}$ On the other hand, stromal calcifications were seen in $90 \%$ of our malignant thyroid nodules, which underscores the standing of stromal calcifications as a common finding in pediatric PTC nodules. The preponderance of stromal calcifications in our pediatric population is larger than the reported range in adults of $47 \%-48 \%$ and is at odds with the previously reported association between these calcifications and advanced age. ${ }^{6,7}$

The retrospective nature and small sample size were restrictions of our study. Additionally, the study was confined to evaluation of echogenic foci, and the influence of other nodule features to assess malignancy risk, including shape, margin, echogenicity, and composition, was not studied. ${ }^{3,4}$ Finally, the pathologic examination of the nodules involved selective sampling. This is an inherent limitation of the histologic examination technique because even if the entire nodule is sampled, only the most superficial sections of the tissue block are examined routinely. Therefore, there may have been unrecognized calcifications or sticky colloid within the tissue blocks, resulting in underestimation of both the presence and distribution.

\section{CONCLUSIONS}

Our study shows that sonographically detectable echogenic foci in malignant pediatric thyroid nodules can be reliably classified into morphologic patterns on the basis of ACR TI-RADS descriptions, with punctate echogenic foci being the most common subtype. Contrary to general knowledge, sonographically detectable echogenic foci within pediatric thyroid carcinoma do not correspond to histopathologic findings of psammomatous calcifications most of the time; instead, more than half of the nodules contained stromal calcifications or sticky colloid. This was true for all types of thyroid malignancy, not only for PTC. These results argue against using the presence of echogenic foci alone as a predictor of malignancy in pediatric thyroid nodules. Rather, when determining of the level of suspicion for malignancy, one should also consider the subtype and the presence of echogenic foci in the context of other features such as margin irregularity and solid composition.

\section{REFERENCES}

1. Francis GL, Waguespack SG, Bauer AJ, et al; American Thyroid Association Guidelines Task Force. Management Guidelines for Children with Thyroid Nodules and Differentiated Thyroid Cancer. Thyroid 2015;25:716-59 CrossRef Medline

2. Haugen B, Alexander EK, Bible KC, et al. 2015 American Thyroid Association Management Guidelines for Adult Patients with Thyroid Nodules and Differentiated Thyroid Cancer: the American Thyroid Association Guidelines Task Force on Thyroid Nodules and Differentiated Thyroid Cancer. Thyroid 2016;26:1133 CrossRef Medline

3. Lim-Dunham JE, Erdem Toslak I, Alsabban K, et al. Ultrasound risk stratification for malignancy using the 2015 American Thyroid Association Management Guidelines for Children with Thyroid Nodules and Differentiated Thyroid Cancer. Pediatr Radiol 2017;47: 429-36 CrossRef Medline

4. Grant EG, Tessler FN, Hoang JK, et al. Thyroid ultrasound reporting lexicon: white paper of the ACR Thyroid Imaging, Reporting and Data System (TIRADS) committee. J Am Coll Radiol 2015;12: 1272-79 CrossRef Medline

5. Miller TR, Bottles K, Holly EA, et al. A step-wise logistic regression analysis of papillary carcinoma of the thyroid. Acta Cytol 1986;30: 285-93 Medline

6. Bai Y, Zhou G, Nakamura M, et al. Survival impact of psammoma body, stromal calcification, and bone formation in papillary thyroid carcinoma. Mod Pathol 2009;22:887-94 CrossRef Medline 
7. Tahvildari AM, Pan L, Kong CS, et al. Sonographic-pathologic correlation for punctate echogenic reflectors in papillary thyroid carcinoma: what are they? J Ultrasound Med 2016;35:1645-52 CrossRef Medline

8. Moon WJ, Jung SL, Lee JH, et al; Thyroid Study Group, Korean Society of Neuro- and Head and Neck Radiology. Benign and malignant thyroid nodules: US differentiation-multicenter retrospective study. Radiology 2008;247:762-70 CrossRef Medline

9. Khoo ML, Asa SL, Witterick IJ, et al. Thyroid calcification and its association with thyroid carcinoma. Head Neck 2002;24:651-55 CrossRef Medline

10. Moudgil P, Vellody R, Heider A, et al. Ultrasound-guided fine-needle aspiration biopsy of pediatric thyroid nodules. Pediatr Radiol 2016;46:365-71 CrossRef Medline

11. Beland MD, Kwon L, Delellis R a, et al. Nonshadowing echogenic foci in thyroid nodules: are certain appearances enough to avoid thyroid biopsy? J Ultrasound Med 2011;30:753-60 CrossRef Medline

12. Malhi H, Beland MD, Cen SY, et al. Echogenic foci in thyroid nodules: significance of posterior acoustic artifacts. AJR Am J Roentgenol 2014;203:1310-16 CrossRef Medline

13. Tessler FN, Middleton WD, Grant EG, et al. ACR Thyroid Imaging, Reporting and Data System (TI-RADS): white paper of the ACR TIRADS Committee. J Am Coll Radiol 2017;14:587-95 CrossRef Medline

14. Cibas ES, Ali SZ. The Bethesda System for Reporting Thyroid Cytopathology. Am J Clin Pathol 2009;132:658-65 CrossRef Medline

15. Muradali D, Colgan T, Hayeems E, et al. Echogenic ovarian foci without shadowing: are they caused by psammomatous calcifications? Radiology 2002;224:429-35 CrossRef Medline

16. Karnak I, Ardiçli B, Ekinci S, et al. Papillary thyroid carcinoma does not have standard course in children. Pediatr Surg Int 2011;27: 931-36 CrossRef Medline 